\title{
Temporal rainfall trend analysis in different agro-ecological regions of southern Africa
}

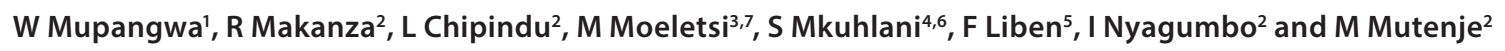 \\ 'International Maize and Wheat Improvement Centre, ILRI Sholla Campus, P O Box 5689, Addis Ababa, Ethiopia \\ ${ }^{2}$ International Maize and Wheat Improvement Centre, PO Box MP 163, Mount Pleasant, Harare, Zimbabwe \\ ${ }^{3}$ Agricultural Research Council, Institute for Soil, Climate and Water, Private Bag X79, Pretoria 0001, South Africa \\ ${ }^{4}$ Climate Systems Analysis Group, Department of Environmental and Geographical Science, University of Cape Town, \\ Rondebosch, South Africa \\ ${ }^{5}$ Department of Agronomy and Horticulture, University of Nebraska-Lincoln, Nebraska, USA \\ 'International Institute for Tropical Agriculture (IITA) C/O ICIPE, PO Box 30772-00100, Nairobi, Kenya \\ ${ }^{7}$ Risk and Vulnerability Science Centre, University of Limpopo, Private Bag X1106, Sovenga, Polokwane 0727, South Africa
}

Rainfall is a major driver of food production in rainfed smallholder farming systems. This study was conducted to assess linear trends in (i) different daily rainfall amounts $\left(<5,5-10,11-20,21-40\right.$ and $\left.>40 \mathrm{~mm} \cdot \mathrm{day}^{-1}\right)$, and (ii) monthly and seasonal rainfall amounts. Drought was determined using the rainfall variability index. Daily rainfall data were derived from 18 meteorological stations in southern Africa. Daily rainfall was dominated by $<5 \mathrm{~mm} \cdot$ day $^{-1}$ followed by 5-10 mm $\cdot$ day $^{-1}$. Three locations experienced increasing linear trends of $<5 \mathrm{~mm} \cdot$ day $^{-1}$ amounts and two others in sub-humid region had increases in the $>40 \mathrm{~mm}^{-1} \mathrm{y}^{-1}$ category. Semi-arid location experienced increasing trends in $<5$ and $5-10 \mathrm{~mm} \cdot$ day $^{-1}$ events. A significant linear trend in seasonal rainfall occurred at two locations with decreasing rainfall $\left(1.24\right.$ and $\left.3 \mathrm{~mm} \cdot \mathrm{season}^{-1}\right)$. A $3 \mathrm{~mm} \cdot \mathrm{season}^{-1}$ decrease in seasonal rainfall was experienced under semi-arid conditions. There were no apparent linear trends in monthly and seasonal rainfall at 15 of the 18 locations studied. Drought frequencies varied with location and were $50 \%$ or higher during the November-March growing season. Rainfall trends were location and agro-ecology specific, but most of the locations studied did not experience significant changes between the $1900 \mathrm{~s}$ and $2000 \mathrm{~s}$.

\section{INTRODUCTION}

Smallholder farming systems in sub-Saharan Africa (SSA) are threatened by climate change and variability and face a huge challenge of producing enough food for close to a billion people in the region (Sonwa et al., 2017). The situation is critical in southern Africa because the region is one of the climate-change hotspots as indicated by recent projections (Lobell et al., 2008; Maure et al., 2018). Rainfall projections indicate mixed trends where some parts of the region will experience no significant changes while rainfall decrease is expected in others (Shongwe et al., 2009; Nicholson et al., 2014; Conway et al., 2015). Approximately 41 million people are already food insecure in southern Africa, the majority of whom are in rural communities that depend on rainfed agriculture (SADC, 2016). The food availability situation is further exacerbated by the continued decline in yields of major cereals and pulses due to a plethora of reasons, including high variability in the start and end of growing seasons, intra-seasonal dry spells, deteriorating soil health and limited use of mineral fertilizers, among others (Cooper et al., 2008; Sileshi et al., 2009; Van Ittersum et al., 2013). Production of major food crops has also been constrained by inappropriate policy environments that do not promote conducive inputoutput markets and producer prices for the smallholder farmers (Smale et al., 2011).

Rainfall is a major driver of crop and livestock production in SSA, with the majority of smallholder agriculture relying on its seasonal amount and distribution (Zinyengere et al., 2011; Mamombe et al., 2017). Smallholder agriculture is dependent on seasonal rainfall because irrigation is generally limited due to poor infrastructure and dwindling water sources (Fanadzo and Ncube, 2018). Major droughts have intensified over time and the current trends show increased frequency in southern Africa (Manatsa et al., 2008; Masih et al., 2014). Compared to seasonal totals, rainfall distribution during the growing season currently has a greater impact on crop and livestock productivity on smallholder farms of southern Africa (Twomlow et al., 2006). The start and the end of the growing season is highly variable (Usman and Reason, 2004; Tadross et al., 2005), making selection of crop types and varieties, and crop establishment methods in the field, difficult for smallholder farmers (Mupangwa et al., 2011; Nyagumbo et al., 2017). Intra-seasonal dry spells are a common feature in the region and often coincide with flowering and early reproductive growth stages of major cereal crops (Usman and Reason, 2004; Cooper et al., 2008). These dry spells significantly reduce yield of the major food security crops and limit biomass production for livestock feed (Ogenga et al., 2018). The high variability in the start and end of rains not only affects food and forage crop productivity on the smallholder farms, but also for communal grazing lands which are critical for livestock production (Manyawu et al., 2016).

Studies from SSA have reported daily rainfall dominated by amounts of less than $10 \mathrm{~mm} \cdot \mathrm{day}^{-1}$ and these have limited impact on crop growth and development (Dixit et al., 2011; Goenster et al., 2015). In Sudan, Goenster et al. (2015) observed that daily rainfall amounts of less than $3 \mathrm{~mm}$ have increased, while 10-20 mm events have declined, since 1970. In southern Africa where daily
CORRESPONDENCE

W Mupangwa

\section{EMAIL}

w.mupangwa@cgiar.org mupangwa@yahoo.com

\section{DATES}

Received: 2 September 2020

Accepted: 24 September 2021

\section{KEYWORDS}

agriculture risk drought rainfall distribution rainfed agriculture

\section{COPYRIGHT}

() The Author(s) Published under a Creative Commons Attribution 4.0 International Licence (CC BY 4.0) 
pan evaporation averages 5-10 $\mathrm{mm} \cdot \mathrm{day}^{-1}$ (Woltering, 2005) and atmospheric evaporative demand is $1.5-10$ times the annual total rainfall (Barron, 2004), rainfall amounts of less than $5 \mathrm{~mm} \cdot \mathrm{day}^{-1}$ have limited impact on crop productivity on smallholder farms where soil water conservation techniques are rarely part of the cropping systems. Clay and sandy soils often require $20-25 \mathrm{~mm}$ and $30-50 \mathrm{~mm}$ of rainfall, respectively, to fully wet the top $30 \mathrm{~cm}$ of the soil profile (Twomlow, 1994; Twomlow and Bruneau, 2000). During the growing season, high intensity storms $\left(>40 \mathrm{~mm} \cdot \mathrm{day}^{-1}\right)$ with high erosivity occur frequently in some parts of southern Africa (Love et al., 2010), leading to reduced water infiltration due to capping and surface sealing in certain soil types and widespread soil erosion on farmlands (Elwell and Stocking, 1988; Twomlow et al., 2006). Additionally, rainfall conditions in southern Africa are already conducive for emerging pests such as the fall armyworm (Spodoptera frugiperda (Smith)) and further variability might worsen pest and disease pressure on smallholder farms (Prasanna et al., 2018). Consequently, household food security in southern Africa remains under threat in the coming decades.

Information on the trends in elements of the weather that drive rainfed farming is critical for decision making by smallholder farmers, agricultural extension agents and research practitioners, rural development agents, the private sector involved in agriculture insurance, and national policy makers. This study was undertaken to assess the trends in daily, monthly and seasonal rainfall over the past decades in selected locations of southern Africa. It was hypothesized that (i) daily rainfall amounts are dominated by light showers $\left(<5 \mathrm{~mm} \cdot \mathrm{day}^{-1}\right)$, (ii) there are linear trends in daily rainfall amounts, and monthly and seasonal rainfall totals, and (iii) seasonal rainfall variability leading to droughts exists in most parts of southern Africa. The specific objectives were to determine: (i) the frequency and assess linear trends in different rainfall classes $(<5,5-10,11-20$, $21-40,>40 \mathrm{~mm} \cdot \mathrm{day}^{-1}$ ), (ii) trends in monthly and seasonal total rainfall, and (iii) drought occurrences in selected locations under different agro-ecological conditions of southern Africa.

\section{MATERIALS AND METHODS}

\section{Data source and quality}

Initial daily rainfall data were collected from 23 meteorological stations spread to cover different agro-ecological conditions of the selected southern African countries (Malawi, Mozambique, South Africa and Zimbabwe). The final daily rainfall data used in the analyses were derived from 18 meteorological stations located in the 4 countries (Appendix, Table A1; Fig. 1). The choice of these stations was based on availability of complete long-term measured/ observed daily rainfall data. Any meteorological station that had missing measured daily rainfall data and needed data infilling at daily, monthly or seasonal timesteps considered in this study, was not included in the analyses, hence reducing the number from 23 to 18 locations. For the final 18 stations, only periods with at least 30 years of data without any missing data were considered, and are summarized in Table A1. The choice of these criteria was based on the availability of data which is considered to be longterm enough for valid trend results in climate change research (Burn and Elnur, 2002). The length of available rainfall datasets varied from country to country and station to station.

Analyses were conducted at daily, monthly and seasonal timesteps in order to answer the research questions selected for the study. Acquired data were converted to the standard June-July calendar (agriculture year in southern Africa) and underwent data quality control routines to identify missing data, errors and suspect data, as well as to ensure that data were consistent and met the data quality objectives. The quality checks were performed using the computer program RClimDex 1.1 and its software package RHtestV3 (Wang and Feng, 2013), that can be accessed at: http://etccdi.pacificclimate.org.

\section{Data analyses}

Time series of daily, monthly and seasonal rainfall data were used to identify trends at different temporal resolutions. Seasonal rainfall totals were used to assess drought occurrences at locations in different agro-ecological regions of Malawi, Mozambique, South Africa and Zimbabwe. Daily rainfall amounts were divided into classes of $<5,5-10,11-20,21-40$ and $>40 \mathrm{~mm} \cdot$ day $^{-1}$.

\section{Mann-Kendall (MK) test}

Non-parametric statistical methods were used to detect temporal linear trends in the daily, monthly and seasonal rainfall data. The main advantages of non-parametric methods are that datasets with missing values are allowed and the data need not conform to any particular distribution (Da Silva at al., 2015). The MannKendall test analysis was performed in $\mathrm{R}$ version 3.5.2 ( $\mathrm{R}$ Core Team, 2018) using the Kendall package to detect the existence of monotonic trends for daily, monthly and seasonal rainfall during the summer season (November-March). The test compares a data value $\left(x_{i}\right.$ and $\left.y_{i}\right)$ to its subsequent values $\left(x_{j}\right.$ and $\left.y_{j}\right)$ and adds an

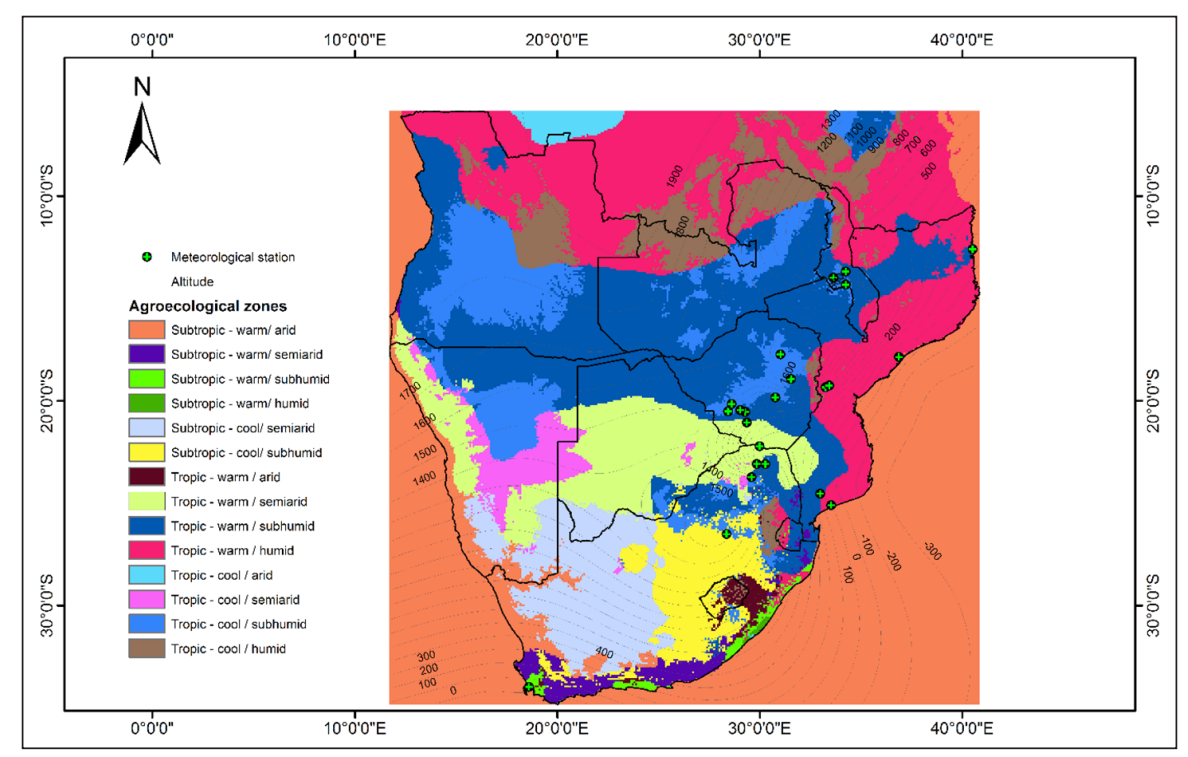

Figure 1. Location of selected meteorological stations in Malawi, Mozambique, South Africa and Zimbabwe used in the study. Different agro-ecological regions of southern Africa are indicated by different colour codes. 
increment or decrement of 1 to the MK test statistic $(S)$ when the subsequent data values were higher or lower, respectively (Karmeshu, 2012), as illustrated in Eq: 1 below. Any missing values were automatically removed during analysis.

$$
S=\sum_{i<j}\left(\operatorname{sign}\left(x_{j}-x_{i}\right) x \operatorname{sign}\left(y_{j}-y_{i}\right)\right)
$$

When there were no ties between the $x$ and $y$ variables, the strength of monotonic association was given by Kendall's rank correlation, tau ( $\tau$ ) (Eq. 2) and subsequent $p$-value of tau for the null hypothesis of no association was calculated (Best and Gipps, 1974).

$$
\tau=S / D \text { where } D=n(n-1) / 2
$$

In the presence of less extensive ties, a normal approximation of $S$ with subsequent continuity correction was made with mean zero, and variance $\operatorname{var}(S)$ where $\operatorname{var}(S)$ was given by Kendall (1975, Eq. 4.4, p. 55).

\section{The Theil-Sen slope estimator}

Since a monotonic trend was demonstrated from the MannKendall test which appeared linear in some stations, the TheilSen slope test was further performed to examine the magnitude of the slope for daily, monthly and seasonal rainfall. The test was performed in R version 3.5.2 (R Core Team, 2018) using the Trend package (Pohlert, 2018). The test computes slope using Sen's method, which calculates a set of linear slopes, followed by a median of the slopes, as follows:

$$
d_{k}=\frac{X_{j}-X_{i}}{j-i}
$$

for $(1 \leq i<j \leq n)$, where $d$ is the slope, $X$ denotes the variable, $n$ is the number of data points, and $i, j$ are indices. As a result, the Sen slope $\left(b_{\text {Sen }}\right)$ is given by $b_{\text {Sen }}=$ median $d_{k}$

\section{Rainfall variability index}

Rainfall variability index $(\delta)$ is calculated as:

$$
\delta_{i}=\left(P_{i}-\mu\right) / \sigma
$$

where $\delta_{i}=$ rainfall variability index for year $i, P_{i}=$ seasonal rainfall for year $i, \mu$ and $\sigma$ are the mean seasonal rainfall and standard deviation for the period under consideration.

In this study $P_{i}$ represented the November-March seasonal rainfall; consequently, $\mu$ and $\sigma$ were the mean and standard deviation of the total seasonal rainfall. A drought year occurs if the $\delta$ is negative and, according to WMO (1975), rainfall timeseries can be classified into different climatic regimes (Table 1). All calculations for the different rainfall ranges were performed in Microsoft Excel.

\section{RESULTS}

\section{Frequency and trends of different daily rainfall amounts}

Daily rainfall events occurring in southern Africa were dominated by amounts of $<5 \mathrm{~mm}$ at all locations (Table 2). Generally, the chances of getting higher daily rainfall amounts decreased consistently across the rainfall classes, regardless of agro-ecological conditions. In Zimbabwe, 5-10 mm.day ${ }^{-1}$, and 11-20 mm.day ${ }^{-1}$ amounts occurred more frequently in sub-humid locations than semi-arid sites. Beitbridge had the least chance (1.4\%) of getting more than $40 \mathrm{~mm} \cdot \mathrm{day}^{-1}$ during the growing season. In Malawi, 21-40 mm.day ${ }^{-1}$ amounts occurred more frequently along Lake Malawi than at further inland locations. Chitedze and Dedza had better chances of getting 11-20 mm.day ${ }^{-1}$ than 5-10 mm.day ${ }^{-1}$ during the growing season. In Mozambique, rainfall amounts of $<5 \mathrm{~mm} \cdot$ day $^{-1}$ occurred more frequently at Chimoio compared to the other locations. In South Africa 5-10 mm.day ${ }^{-1}$ events occurred more frequently than $<5 \mathrm{~mm} \cdot$ day $^{-1}$ at Harmony.

Table 1. Rainfall ranges and corresponding climatic regimes based on WMO classification (Source: WMO, 1975)

\begin{tabular}{|c|c|c|c|c|c|c|}
\hline \multirow[t]{3}{*}{ Country } & \multirow[t]{3}{*}{ Station } & \multicolumn{5}{|c|}{ Rainfall amount $\left(\mathrm{mm} \cdot\right.$ day $\left.^{-1}\right)$} \\
\hline & & $<5$ & $5-10$ & $11-20$ & $21-40$ & $>40$ \\
\hline & & \multicolumn{5}{|c|}{ Probability (\%) } \\
\hline \multirow[t]{6}{*}{ Zimbabwe } & Harare & 22.5 & 11.8 & 12.7 & 8.0 & 2.9 \\
\hline & Marondera & 20.5 & 12.0 & 11.9 & 8.5 & 3.3 \\
\hline & Matopos & 13.2 & 7.5 & 8.0 & 6.3 & 1.9 \\
\hline & Bulawayo & 16.1 & 8.8 & 8.6 & 6.1 & 2.0 \\
\hline & W. Nich. & 16.5 & 6.5 & 5.8 & 4.4 & 1.8 \\
\hline & Beitbridge & 13.1 & 5.3 & 4.7 & 3.1 & 1.3 \\
\hline \multirow[t]{3}{*}{ Malawi } & Chitala & 17.4 & 12.5 & 12.3 & 10.4 & 4.0 \\
\hline & Chitedze & 23.1 & 13.3 & 14.6 & 8.9 & 3.8 \\
\hline & Dedza & 26.1 & 14.8 & 15.5 & 9.7 & 3.3 \\
\hline \multirow[t]{5}{*}{ Mozambique } & Chimoio & 21.3 & 11.2 & 11.2 & 8.6 & 5.4 \\
\hline & Chokwe & 14.7 & 6.5 & 5.0 & 3.9 & 2.1 \\
\hline & Pemba & 17.8 & 9.1 & 9.3 & 6.7 & 3.6 \\
\hline & Quelimane & 17.9 & 9.2 & 9.8 & 8.6 & 6.6 \\
\hline & Xai Xai & 19.4 & 7.2 & 6.3 & 5.0 & 3.4 \\
\hline \multirow[t]{4}{*}{ South Africa } & Harmony & 10.8 & 12.5 & 6.8 & 4.0 & 1.8 \\
\hline & Levubu & 22.0 & 8.3 & 7.7 & 6.3 & 5.0 \\
\hline & Mertz & 10.5 & 10.3 & 7.1 & 3.8 & 1.8 \\
\hline & Polokwane & 15.1 & 7.3 & 6.2 & 4.1 & 1.4 \\
\hline
\end{tabular}

\begin{tabular}{lc}
\hline Rainfall range & Climatic regime \\
\hline$P<\mu-2 \cdot \sigma$ & Extremely dry \\
$\mu-2 \cdot \sigma<P<\mu-\sigma$ & Dry \\
$\mu-\sigma<P<\mu+\sigma$ & Normal \\
$P>\mu+\sigma$ & Wet \\
\hline
\end{tabular}

Table 2. The frequency (\%) of receiving different amount during the November-March growing season at different weather stations in Zimbabwe, Malawi, Mozambique and South Africa. 
Trends of the different rainfall classes varied with location (Tables 3 and 4). Significant increases in the $>40 \mathrm{~mm} \cdot \mathrm{day}^{-1}$ class were detected at one of the 6 locations in Zimbabwe. Beitbridge experienced an increasing trajectory in $<5$ and $5-10 \mathrm{~mm} \cdot$ day $^{-1}$ amounts. A significantly decreasing trajectory of $<5$ day $^{-1}$ amounts was detected at one of the 6 Zimbabwean locations. Chitala and
Chitedze experienced decreases in $<5$ and $5-10 \mathrm{~mm} \cdot$ day $^{-1}$, and increases in $<5 \mathrm{~mm} \cdot$ day $^{-1}$ rainfall amounts, respectively. Significant decreases in $<5$ and $11-20 \mathrm{~mm} \cdot$ day $^{-1}$, and $5-10 \mathrm{~mm} \cdot$ day $^{-1}$ amounts were detected at Chimoio and Chokwe, respectively (Table 5). The $>40$ and 11-20 mm.day ${ }^{-1}$ amounts decreased over time at Harmony and Mertz, respectively (Table 6).

Table 3. Mann-Kendall trend and Sen slope for different rainfall classes at weather stations in Zimbabwe

\begin{tabular}{|c|c|c|c|c|c|}
\hline Station & Rainfall class & Kendall's tau & $P$-value & Sen slope & $P$-value \\
\hline \multirow[t]{5}{*}{ Harare } & $<5$ & 0.0247 & 0.8367 & 0.0000 & 0.8367 \\
\hline & $5-10$ & 0.0125 & 0.9226 & 0.0000 & 0.9226 \\
\hline & $11-20$ & 0.0961 & 0.4084 & 0.0625 & 0.4084 \\
\hline & $21-40$ & -0.0310 & 0.7979 & 0.0000 & 0.7979 \\
\hline & $>40$ & 0.2287 & 0.0577 & 0.0400 & 0.0557 \\
\hline \multirow[t]{5}{*}{ Marondera } & $<5$ & -0.2937 & 0.0036 & -0.200 & 0.0036 \\
\hline & $5-10$ & -0.1770 & 0.0807 & -0.1026 & 0.0807 \\
\hline & $11-20$ & -0.1302 & 0.2004 & -0.0659 & 0.2004 \\
\hline & $21-40$ & 0.1053 & 0.3061 & 0.0345 & 0.3061 \\
\hline & $>40$ & -0.0792 & 0.4557 & 0.0000 & 0.4557 \\
\hline \multirow[t]{5}{*}{ Matopos } & $<5$ & -0.0956 & 0.2335 & -0.0396 & 0.2335 \\
\hline & $5-10$ & -0.1720 & 0.0344 & -0.0367 & 0.0344 \\
\hline & $11-20$ & -0.1717 & 0.0335 & -0.0440 & 0.0335 \\
\hline & $21-40$ & -0.0978 & 0.2327 & 0.0000 & 0.2327 \\
\hline & $>40$ & -0.0111 & 0.9007 & 0.0000 & 0.9007 \\
\hline \multirow[t]{5}{*}{ Bulawayo } & $<5$ & 0.0698 & 0.4030 & 0.0222 & 0.4030 \\
\hline & $5-10$ & -0.1417 & 0.0941 & -0.0263 & 0.0941 \\
\hline & $11-20$ & 0.0462 & 0.5834 & 0.0000 & 0.5834 \\
\hline & $21-40$ & -0.0183 & 0.8333 & 0.0000 & 0.8333 \\
\hline & $>40$ & 0.0169 & 0.8509 & 0.0000 & 0.8509 \\
\hline \multirow[t]{5}{*}{ West Nich } & $<5$ & 0.1022 & 0.3756 & 0.0800 & 0.3756 \\
\hline & $5-10$ & -0.0700 & 0.9612 & 0.0000 & 0.9612 \\
\hline & $11-20$ & -0.0958 & 0.4140 & -0.0345 & 0.4140 \\
\hline & $21-40$ & 0.0904 & 0.4473 & 0.0000 & 0.4473 \\
\hline & $>40$ & 0.0699 & 0.5684 & 0.0000 & 0.5684 \\
\hline \multirow[t]{5}{*}{ Beitbridge } & $<5$ & 0.2396 & 0.0181 & 0.1429 & 0.0181 \\
\hline & $5-10$ & 0.0238 & 0.0212 & 0.0667 & 0.0212 \\
\hline & $11-20$ & 0.1902 & 0.0643 & 0.0606 & 0.0643 \\
\hline & $21-40$ & 0.1770 & 0.0954 & 0.0000 & 0.0954 \\
\hline & $>40$ & -0.0558 & 0.6175 & 0.0000 & 0.6175 \\
\hline
\end{tabular}

Table 4. Mann-Kendall trend and Sen slope for different rainfall classes at weather stations in Malawi

\begin{tabular}{|c|c|c|c|c|c|}
\hline Station & Rainfall class & Kendall's tau & $P$-value & Sen slope & $P$-value \\
\hline \multirow[t]{5}{*}{ Chitala } & $<5$ & -0.0882 & 0.3745 & -0.0278 & 0.3745 \\
\hline & $5-10$ & -0.3657 & 0.0002 & -0.1567 & 0.0002 \\
\hline & $11-20$ & -0.0976 & 0.3303 & 0.0000 & 0.3303 \\
\hline & $21-40$ & 0.0755 & 0.4506 & 0.0000 & 0.4506 \\
\hline & $>40$ & 0.0489 & 0.6353 & 0.0000 & 0.6353 \\
\hline \multirow[t]{5}{*}{ Chitedze } & $<5$ & 0.2864 & 0.0230 & 0.0262 & 0.0230 \\
\hline & $5-10$ & -0.0176 & 0.9008 & 0.0000 & 0.9008 \\
\hline & $11-20$ & 0.0938 & 0.4638 & 0.0625 & 0.4638 \\
\hline & $21-40$ & 0.0885 & 0.5194 & 0.0000 & 0.5194 \\
\hline & $>40$ & -0.1816 & 0.1655 & -0.0465 & 0.1655 \\
\hline \multirow[t]{5}{*}{ Dedza } & $<5$ & -0.1556 & 0.1575 & -0.1053 & 0.1575 \\
\hline & $5-10$ & -0.1325 & 0.2313 & -0.0690 & 0.2313 \\
\hline & $11-20$ & 0.0598 & 0.9653 & 0.0000 & 0.9653 \\
\hline & $21-40$ & 0.1649 & 0.1406 & 0.0625 & 0.1406 \\
\hline & $>40$ & -0.1742 & 0.1389 & 0.0000 & 0.1389 \\
\hline
\end{tabular}


Table 5. Mann-Kendall trend test of different rainfall classes at weather stations in Mozambique

\begin{tabular}{|c|c|c|c|c|c|c|}
\hline Country & Station & Rainfall class & Kendall's tau & $P$-value & Sen slope & $P$-value \\
\hline \multirow[t]{25}{*}{ Mozambique } & Chimoio & $<5$ & -0.1609 & 0.0722 & -0.0714 & 0.0722 \\
\hline & & $5-10$ & -0.1062 & 0.2388 & -0.0357 & 0.2388 \\
\hline & & $11-20$ & -0.1950 & 0.0295 & -0.0769 & 0.0295 \\
\hline & & $21-40$ & -0.0055 & 0.9562 & 0.0000 & 0.9562 \\
\hline & & $>40$ & -0.0269 & 0.7732 & 0.0000 & 0.7732 \\
\hline & Chokwe & $<5$ & -0.0104 & 0.9431 & 0.0000 & 0.9431 \\
\hline & & $5-10$ & -0.2707 & 0.0292 & -0.0952 & 0.0292 \\
\hline & & $11-20$ & 0.2403 & 0.0567 & 0.0606 & 0.0567 \\
\hline & & $21-40$ & 0.0432 & 0.7401 & 0.0000 & 0.7401 \\
\hline & & $>40$ & -0.2177 & 0.0889 & -0.0370 & 0.0889 \\
\hline & Quelimane & $<5$ & -0.0671 & 0.5155 & 0.0000 & 0.5155 \\
\hline & & $5-10$ & -0.0798 & 0.4401 & 0.0000 & 0.4401 \\
\hline & & $11-20$ & 0.0505 & 0.6270 & 0.0000 & 0.6270 \\
\hline & & $21-40$ & -0.0142 & 0.8965 & 0.0000 & 0.8965 \\
\hline & & $>40$ & -0.0876 & 0.3990 & 0.0000 & 0.3990 \\
\hline & Pemba & $<5$ & -0.0700 & 0.4661 & -0.0256 & 0.4661 \\
\hline & & $5-10$ & -0.0237 & 0.8098 & 0.0000 & 0.8098 \\
\hline & & $11-20$ & 0.1865 & 0.0529 & 0.0513 & 0.0529 \\
\hline & & $21-40$ & 0.0204 & 0.8378 & 0.0000 & 0.8378 \\
\hline & & $>40$ & 0.0836 & 0.4052 & 0.0000 & 0.4052 \\
\hline & Xai Xai & $<5$ & -0.0570 & 0.6315 & -0.0370 & 0.6315 \\
\hline & & $5-10$ & -0.0707 & 0.5586 & 0.0000 & 0.5586 \\
\hline & & $11-20$ & -0.0431 & 0.7227 & 0.0000 & 0.7227 \\
\hline & & $21-40$ & 0.0597 & 0.6261 & 0.0000 & 0.6261 \\
\hline & & $>40$ & -0.1438 & 0.2315 & -0.0333 & 0.2315 \\
\hline
\end{tabular}

Table 6. Mann-Kendall trend test of different rainfall classes at weather stations in South Africa

\begin{tabular}{|c|c|c|c|c|c|c|}
\hline Country & Station & Rainfall class & Kendall's tau & $P$-value & Sen slope & $P$-value \\
\hline \multirow[t]{20}{*}{ SA } & Harmony & $<5$ & 0.2667 & 0.0002 & 0.1053 & 0.0002 \\
\hline & & $5-10$ & 0.0351 & 0.6210 & 0.0000 & 0.6210 \\
\hline & & $11-20$ & -0.0887 & 0.2220 & 0.0000 & 0.2220 \\
\hline & & $21-40$ & -0.0720 & 0.3237 & 0.0000 & 0.3237 \\
\hline & & $>40$ & -0.2698 & 0.0004 & -0.0141 & 0.0004 \\
\hline & Levubu & $<5$ & -0.0497 & 0.6709 & -0.0313 & 0.6709 \\
\hline & & $5-10$ & 0.0535 & 0.6521 & 0.0000 & 0.6521 \\
\hline & & $11-20$ & -0.0324 & 0.7884 & 0.0000 & 0.7884 \\
\hline & & $21-40$ & 0.0423 & 0.7234 & 0.0000 & 0.7234 \\
\hline & & $>40$ & -0.0084 & 0.9515 & 0.0000 & 0.9515 \\
\hline & Mertz & $<5$ & -0.1591 & 0.0244 & -0.0606 & 0.0244 \\
\hline & & $5-10$ & -0.3836 & $6.65 e^{-8}$ & -0.1092 & $6.65 e^{-8}$ \\
\hline & & $11-20$ & -0.1756 & 0.0146 & -0.0323 & 0.0146 \\
\hline & & $21-40$ & -0.0401 & 0.5864 & 0.0000 & 0.5864 \\
\hline & & $>40$ & 0.2840 & 0.0002 & 0.0156 & 0.0002 \\
\hline & Polokwane & $<5$ & -0.1454 & 0.1681 & -0.0588 & 0.1681 \\
\hline & & $5-10$ & 0.0440 & 0.6876 & 0.0000 & 0.6876 \\
\hline & & $11-20$ & -0.0446 & 0.6815 & 0.0000 & 0.6815 \\
\hline & & $21-40$ & 0.0186 & 0.8704 & 0.0000 & 0.8704 \\
\hline & & $>40$ & 0.2001 & 0.0803 & 0.0000 & 0.0803 \\
\hline
\end{tabular}

\section{Trends of monthly and seasonal rainfall}

The presence of linear trends in monthly and seasonal (November-March) rainfall varied between locations. The November-March period had significant increasing (0.09 $\mathrm{mm} \cdot$ season $^{-1}$ ) and decreasing rainfall trajectories at Matopos and Beitbridge, respectively (Table 7). January rainfall decreased by $1.8 \mathrm{~mm} \cdot$ season $^{-1}$ while seasonal total declined by $0.3 \mathrm{~mm} \cdot \mathrm{season}^{-1}$ at Beitbridge. The January and March rainfall significantly increased at 3.3 and $1.8 \mathrm{~mm} \cdot \mathrm{season}^{-1}$ at Harare. March and November-March rainfall increased $(P<0.05)$ by 0.6 and
$2.1 \mathrm{~mm} \cdot$ season $^{-1}$, respectively, at Bulawayo. There was a general decrease in February and March rainfall at Malawian locations (Table 8$)$. February rainfall significantly $(P=0.0132)$ decreased by $2.5 \mathrm{~mm} \cdot \mathrm{season}^{-1}$ at Chitala. Rainfall decreased by $0.15-$ $3.7 \mathrm{~mm} \cdot$ season $^{-1}$ during the November-March period at 3 of the 5 locations in Mozambique (Table 9). February rainfall decreased $(P=0.0111)$ by $3.3 \mathrm{~mm} \cdot$ season $^{-1}$ at Xai Xai. In South Africa, February rainfall decreased at three locations (Table 10). During the November-March period, rainfall decreased $(P=0.0421)$ by $1.24 \mathrm{~mm} \cdot$ season $^{-1}$ at Harmony. 
Table 7. Mann-Kendall trend and Sen slope tests of monthly and seasonal (November-March) rainfall at weather stations in Zimbabwe

\begin{tabular}{|c|c|c|c|c|c|c|}
\hline Station & Month(s) & $N$ & Kendall's tau & $P$-value & Sen slope & $P$-value \\
\hline \multirow[t]{6}{*}{ Harare } & Nov & 38 & -0.053 & 0.651 & -0.5429 & 0.6418 \\
\hline & Dec & 38 & -0.027 & 0.821 & -0.2462 & 0.8210 \\
\hline & Jan & 38 & 0.260 & 0.022 & 3.2550 & 0.0221 \\
\hline & Feb & 38 & -0.018 & 0.022 & -0.2320 & 0.8801 \\
\hline & Mar & 38 & 0.240 & 0.035 & 1.7889 & 0.0347 \\
\hline & Nov-Mar & 38 & 0.073 & 0.530 & 2.2273 & 0.5296 \\
\hline \multirow[t]{6}{*}{ Marondera } & Nov & 50 & -0.034 & 0.737 & 0.2292 & 0.3234 \\
\hline & Dec & 50 & -0.160 & 0.107 & 0.2727 & 0.2767 \\
\hline & Jan & 50 & -0.075 & 0.453 & -0.0370 & 0.9067 \\
\hline & Feb & 50 & -0.095 & 0.339 & 0.2286 & 0.4413 \\
\hline & Mar & 50 & 0.153 & 0.123 & 0.1600 & 0.5347 \\
\hline & Nov-Mar & 50 & -0.095 & 0.339 & 0.8929 & 0.3194 \\
\hline \multirow[t]{6}{*}{ Matopos } & Nov & 76 & -0.105 & 0.184 & 0.000 & 0.2884 \\
\hline & Dec & 76 & -0.056 & 0.476 & -0.0910 & 0.8365 \\
\hline & Jan & 76 & -0.094 & 0.231 & 0.0498 & 0.8894 \\
\hline & Feb & 76 & -0.110 & 0.163 & 0.2388 & 0.5068 \\
\hline & Mar & 76 & -0.168 & 0.033 & 0.4493 & 0.0817 \\
\hline & Nov-Mar & 76 & -0.176 & 0.025 & 0.0941 & 0.0517 \\
\hline \multirow[t]{6}{*}{ Bulawayo } & Nov & 71 & 0.084 & 0.302 & 0.5887 & 0.0685 \\
\hline & Dec & 71 & -0.046 & 0.571 & -0.4550 & 0.2770 \\
\hline & Jan & 71 & -0.038 & 0.641 & 0.2866 & 0.5715 \\
\hline & Feb & 71 & 0.002 & 0.984 & 0.7154 & 0.1056 \\
\hline & Mar & 71 & 0.008 & 0.925 & 0.6000 & 0.0110 \\
\hline & Nov-Mar & 71 & 0.012 & 0.889 & 2.0817 & 0.0418 \\
\hline \multirow[t]{6}{*}{ West Nich } & Nov & 39 & -0.004 & 0.981 & -0.0333 & 0.9807 \\
\hline & Dec & 39 & -0.112 & 0.321 & -0.7214 & 0.3212 \\
\hline & Jan & 39 & 0.093 & 0.411 & 0.9182 & 0.4107 \\
\hline & Feb & 39 & -0.007 & 0.961 & -0.0250 & 0.9614 \\
\hline & Mar & 39 & 0.119 & 0.293 & 0.6000 & 0.2926 \\
\hline & Nov-Mar & 39 & 0.026 & 0.828 & 0.4677 & 0.8276 \\
\hline \multirow[t]{6}{*}{ Beitbridge } & Nov & 50 & 0.138 & 0.160 & -0.1875 & 0.5582 \\
\hline & Dec & 50 & -0.026 & 0.795 & -0.0681 & 0.8474 \\
\hline & Jan & 50 & 0.156 & 0.112 & -1.7727 & 0.0052 \\
\hline & Feb & 50 & 0.105 & 0.284 & -0.6429 & 0.2553 \\
\hline & Mar & 50 & 0.180 & 0.035 & -0.2826 & 0.2880 \\
\hline & Nov-Mar & 50 & 0.176 & 0.043 & -0.3000 & 0.0139 \\
\hline
\end{tabular}

Table 8. Mann-Kendall trend test of monthly and seasonal rainfall at weather stations in Malawi

\begin{tabular}{lcccccc}
\hline Station & Month & $\boldsymbol{N}$ & Kendall's tau & $\boldsymbol{P}$-value & Sen slope & $P$-value \\
\hline Chitala & Nov & 52 & -0.0630 & 0.5173 & -0.1467 & 0.5173 \\
& Dec & 52 & 0.1028 & 0.2866 & 0.8475 & 0.2866 \\
Jan & 52 & 0.1086 & 0.2590 & 0.8967 & 0.2591 \\
& Feb & 52 & -0.2382 & 0.0132 & -2.5286 & 0.0132 \\
& Mar & 52 & -0.0045 & 0.9685 & -0.0063 & 0.9685 \\
& Nov-Mar & 52 & -0.0166 & 0.8684 & -0.3896 & 0.8684 \\
\hline Chitedze & Nov & 33 & 0.0000 & 1.0000 & 0.0110 & 1.0000 \\
& Dec & 33 & -0.0057 & 0.9753 & -0.0577 & 0.9753 \\
& Jan & 33 & 0.16330 & 0.1930 & 1.9912 & 0.1930 \\
& Feb & 33 & -0.0909 & 0.4665 & -1.1479 & 0.4665 \\
& Mar & 33 & -0.0719 & 0.5664 & -0.9393 & 0.5664 \\
& Nov-Mar & 33 & 0.0000 & 1.0000 & 0.0110 & 1.0000 \\
\hline Dedza & Nov & 41 & 0.1049 & 0.3397 & 0.5476 & 0.3397 \\
& Dec & 41 & 0.0317 & 0.7789 & 0.2730 & 0.7797 \\
& Jan & 41 & -0.0952 & 0.3871 & -1.2443 & 0.3871 \\
& Feb & 41 & -0.0647 & 0.5592 & -0.6125 & 0.5592 \\
& Mar & 41 & -0.0354 & 0.7531 & -0.3711 & 0.7531 \\
& Nov-Mar & 41 & -0.0195 & 0.8662 & -0.5900 & 0.8662 \\
\hline
\end{tabular}


Table 9. Mann-Kendall trend test of monthly and seasonal rainfall at weather stations in Mozambique

\begin{tabular}{|c|c|c|c|c|c|c|}
\hline Station & Month & $N$ & Kendall's tau & $P$-value & Sen slope & $P$-value \\
\hline \multirow[t]{6}{*}{ Chimoio } & Nov & 62 & 0.0952 & 0.2769 & 0.5000 & 0.2769 \\
\hline & Dec & 62 & -0.0407 & 0.6443 & -0.4357 & 0.6443 \\
\hline & Jan & 62 & 0.0085 & 0.9274 & 0.1333 & 0.9274 \\
\hline & Feb & 62 & 0.0619 & 0.4811 & 0.7103 & 0.4811 \\
\hline & Mar & 62 & 0.0709 & 0.4192 & 0.4864 & 0.4192 \\
\hline & Nov-Mar & 62 & 0.0423 & 0.6313 & 1.1143 & 0.6313 \\
\hline \multirow[t]{6}{*}{ Chokwe } & Nov & 35 & 0.0151 & 0.9095 & 0.0944 & 0.9095 \\
\hline & Dec & 35 & 0.0723 & 0.5509 & 0.5125 & 0.5509 \\
\hline & Jan & 35 & -0.1899 & 0.1117 & -2.1200 & 0.1117 \\
\hline & Feb & 35 & 0.0151 & 0.9095 & 0.1000 & 0.9095 \\
\hline & Mar & 35 & -0.0538 & 0.6597 & -0.2286 & 0.6597 \\
\hline & Nov-Mar & 35 & -0.1261 & 0.2933 & -3.2917 & 0.2933 \\
\hline \multirow[t]{6}{*}{ Quelimane } & Nov & 49 & 0.1192 & 0.2308 & 0.5489 & 0.2308 \\
\hline & Dec & 49 & -0.0459 & 0.6478 & -0.4681 & 0.6478 \\
\hline & Jan & 49 & -0.0799 & 0.4228 & -1.1542 & 0.4228 \\
\hline & Feb & 49 & 0.0204 & 0.8428 & 0.2133 & 0.8428 \\
\hline & Mar & 49 & 0.1225 & 0.2177 & 1.9106 & 0.2177 \\
\hline & Nov-Mar & 49 & 0.0136 & 0.8971 & -0.5482 & 0.8971 \\
\hline \multirow[t]{6}{*}{ Pemba } & Nov & 55 & -0.0866 & 0.3563 & -0.1469 & 0.3563 \\
\hline & Dec & 55 & 0.1447 & 0.1203 & 0.9424 & 0.1203 \\
\hline & Jan & 55 & -0.0842 & 0.3680 & -0.5927 & 0.3680 \\
\hline & Feb & 55 & -0.0074 & 0.9421 & -0.0500 & 0.9421 \\
\hline & Mar & 55 & 0.0303 & 0.7494 & 0.2910 & 0.7494 \\
\hline & Nov-Mar & 55 & 0.0222 & 0.8163 & 0.3333 & 0.8163 \\
\hline \multirow[t]{6}{*}{ Xai Xai } & Nov & 38 & -0.1480 & 0.1953 & -0.7895 & 0.1953 \\
\hline & Dec & 38 & 0.0655 & 0.5715 & 0.5000 & 0.5715 \\
\hline & Jan & 38 & 0.0370 & 0.7533 & 0.3054 & 0.7533 \\
\hline & Feb & 38 & -0.2888 & 0.0111 & -3.3167 & 0.0111 \\
\hline & Mar & 38 & 0.0213 & 0.8603 & 0.1333 & 0.8603 \\
\hline & Nov-Mar & 38 & -0.1607 & 0.1591 & -3.7250 & 0.1591 \\
\hline
\end{tabular}

Table 10. Mann-Kendall trend test of monthly and seasonal rainfall at weather stations in South Africa

\begin{tabular}{|c|c|c|c|c|c|c|}
\hline Station & Month & $N$ & Kendall's tau & $P$-value & Sen slope & $P$-value \\
\hline \multirow[t]{6}{*}{ Harmony } & Nov & 96 & -0.0715 & 0.3036 & -0.1562 & 0.3036 \\
\hline & Dec & 96 & -0.1562 & 0.0244 & -0.4119 & 0.0244 \\
\hline & Jan & 96 & -0.0959 & 0.1676 & -0.2490 & 0.1676 \\
\hline & Feb & 96 & -0.0748 & 0.2818 & -0.2846 & 0.2818 \\
\hline & Mar & 96 & -0.0242 & 0.7301 & -0.0552 & 0.7301 \\
\hline & Nov-Mar & 96 & -0.1410 & 0.0421 & -1.2397 & 0.0421 \\
\hline \multirow[t]{6}{*}{ Levubu } & Nov & 39 & 0.0202 & 0.8655 & 0.1629 & 0.8655 \\
\hline & Dec & 39 & 0.0418 & 0.7167 & 0.3044 & 0.7167 \\
\hline & Jan & 39 & -0.0065 & 0.9614 & -0.1106 & 0.9614 \\
\hline & Feb & 39 & -0.0958 & 0.3971 & -1.1417 & 0.3971 \\
\hline & Mar & 39 & -0.0445 & 0.6987 & -0.3950 & 0.6987 \\
\hline & Nov-Mar & 39 & 0.0122 & 0.9229 & 0.6000 & 0.9229 \\
\hline \multirow[t]{6}{*}{ Mertz } & Nov & 96 & 0.0466 & 0.5041 & 0.1043 & 0.5041 \\
\hline & Dec & 96 & -0.0147 & 0.8345 & -0.0417 & 0.8345 \\
\hline & Jan & 96 & 0.0029 & 0.9697 & 0.0072 & 0.9697 \\
\hline & Feb & 96 & -0.0411 & 0.5560 & -0.1321 & 0.5560 \\
\hline & Mar & 96 & 0.0176 & 0.8025 & 0.0427 & 0.8025 \\
\hline & Nov-Mar & 96 & -0.0075 & 0.9167 & 0.0000 & 0.9167 \\
\hline \multirow[t]{6}{*}{ Polokwane } & Nov & 45 & 0.0657 & 0.5313 & 0.3114 & 0.5313 \\
\hline & Dec & 45 & 0.0172 & 0.8756 & 0.0599 & 0.8756 \\
\hline & Jan & 45 & 0.0000 & 1.0000 & -0.0063 & 1.0000 \\
\hline & Feb & 45 & 0.0424 & 0.6884 & 0.1289 & 0.6884 \\
\hline & Mar & 45 & 0.0788 & 0.4513 & 0.3285 & 0.4513 \\
\hline & Nov-Mar & 45 & 0.1051 & 0.3137 & 1.2479 & 0.3137 \\
\hline
\end{tabular}




\section{Seasonal rainfall variability}

The number of drought years varied with location and the period considered for each station. Harare experienced 20 droughts, with 12 being very dry, and 3 wet years during the 38 -year period (Fig. 2). The worst droughts occurred during 1964, 1968 and 1995. Marondera experienced one extreme drought (1992) and 7 wet years over a 49 -year period; 41 droughts were experienced between 1940 and 2015, and most wet years occurred before 1975 at Matopos. The number of wet years decreased between 1980 to 2015. At Bulawayo, 39 droughts and 13 wet years were experienced. West Nicholson experienced 21 droughts and just 6 wet years in 39 years; 29 droughts in 50 years were experienced at Beitbridge and the 1960 and 1980s were the driest decades with 5 severe droughts occurring. Only 4 wet years were experienced between 1952 and 2001 and this included the El Niño year.

At Chitala, 26 droughts were experienced, evenly distributed over the 52 years, with the majority occurring during the 1980s and 1990s (Fig. 3); 6 wet years were recorded between 1976 and 1986. At Chitedze, 18 droughts were experienced and 6 of them were very dry; 7 dry and 4 wet seasons were experienced at Dedza where a total of 21 droughts were recorded in 33 years.
Chimoio experienced an extremely dry 1972 and the extreme drought of 1972 was immediately followed by 3 consecutive wet years (Fig. 4). A total of 34 droughts occurred at Chimoio in 62 years. At Chokwe, 18 droughts occurred and most of the droughts occurred in the 1980s; 5 wet years, evenly distributed over the 35 years, occurred at Chokwe; and 29 droughts with 1 extreme and 5 wet years were experienced at Pemba in 54 years. The droughts were concentrated in the 1960s and 1970s. Quelimane had 28 droughts, one of them being extreme and 5 wet years were experienced in the 1960s and 1980s. A very dry year followed by a relatively wet one occurred once at Quelimane during the 1960s. At Xai Xai, 24 droughts and 7 wet years were recorded in 38 years.

The Mertz location experienced 58 droughts and 15 wet years over a 96-year period. Most of the droughts occurred in the 1940s, 1980s and 1990s (Fig. 5). At Harmony, 56 droughts, with 8 being very dry, and 9 wet years occurred in 96 years. Most of the droughts occurred between the 1920s and 1940s. Over a 39-year period, 22 droughts and 5 wet years occurred at Levubu. Most of the droughts occurred between 1982 and 2004. In 45 years, 26 droughts and 5 wet years were experienced at Polokwane.
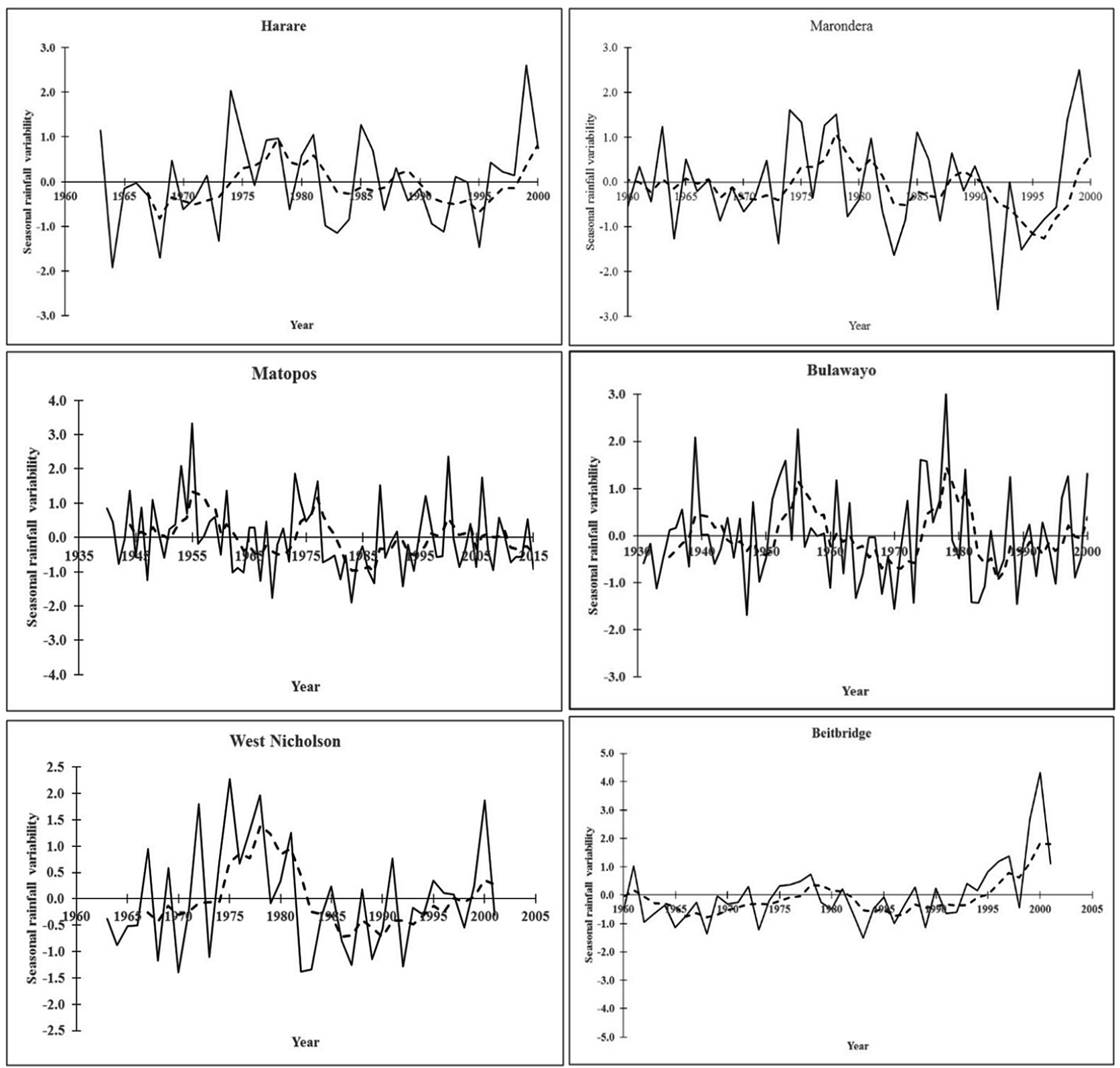

Figure 2. Seasonal rainfall variation at different locations in Zimbabwe. Dotted line represents 5-year moving average. 

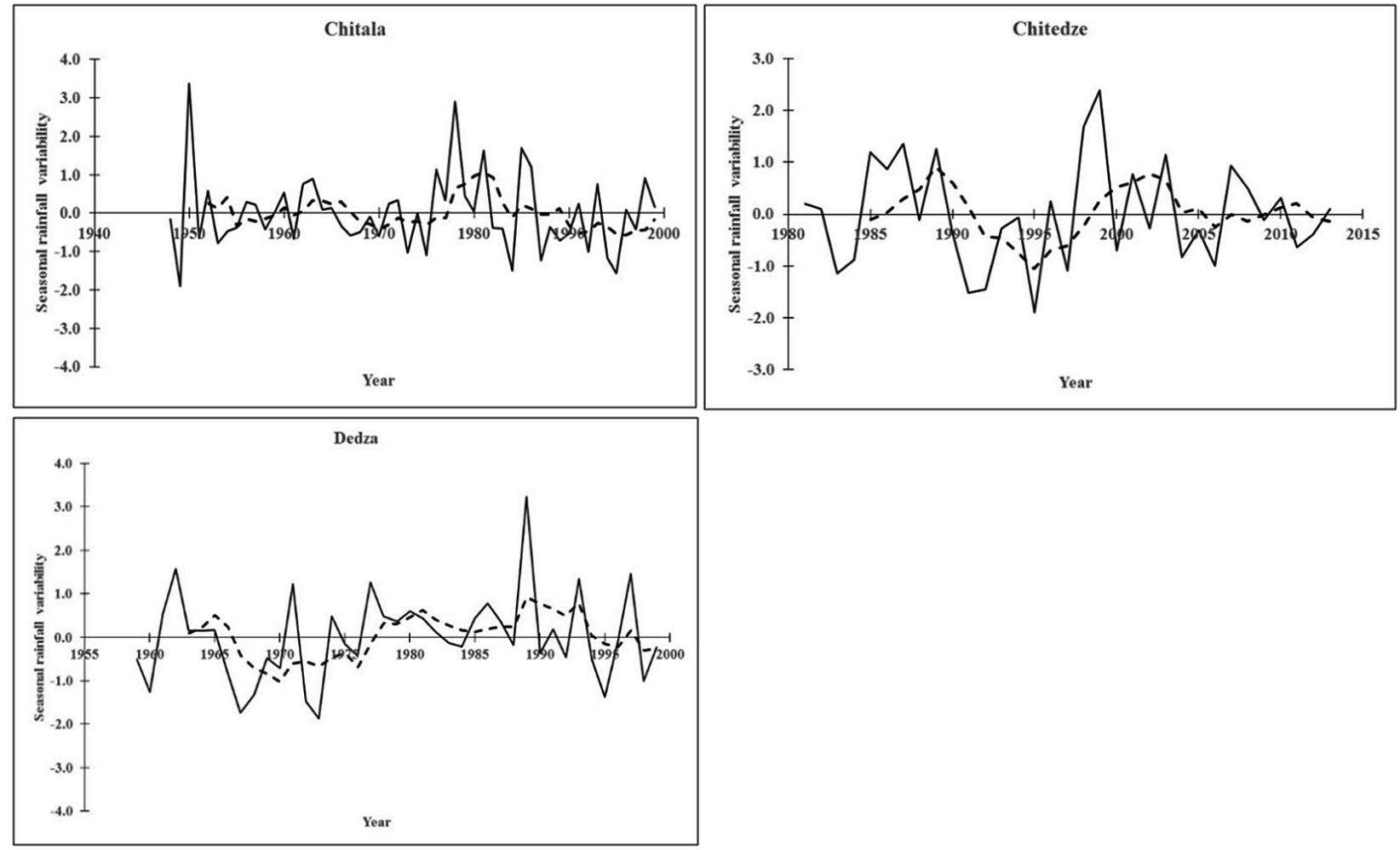

Figure 3. Seasonal rainfall variation at different locations in Malawi. Dotted line represents 5-year moving average.
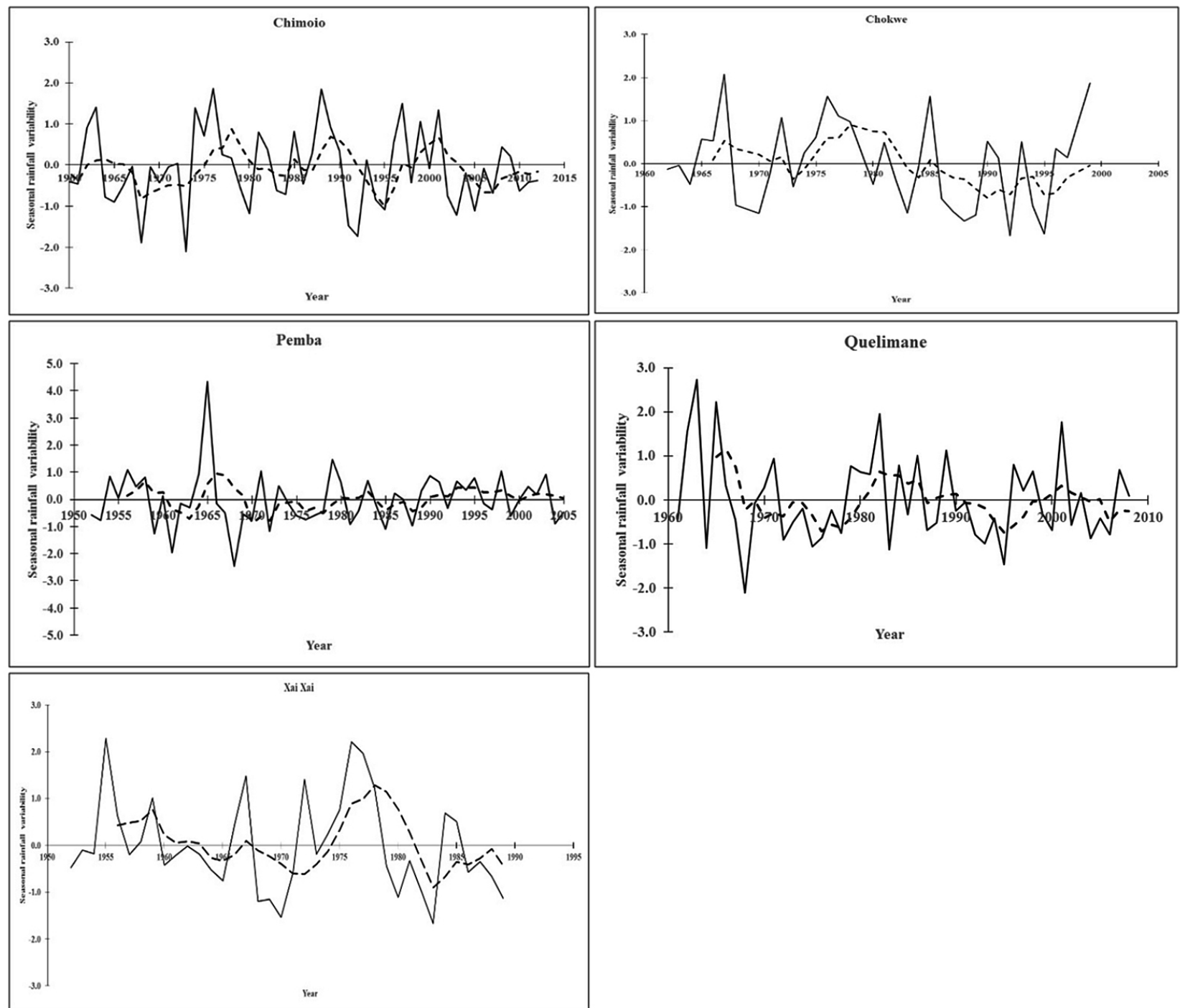

Figure 4. Seasonal rainfall variation at different locations in Mozambique. Dotted line represents 5-year moving average. 

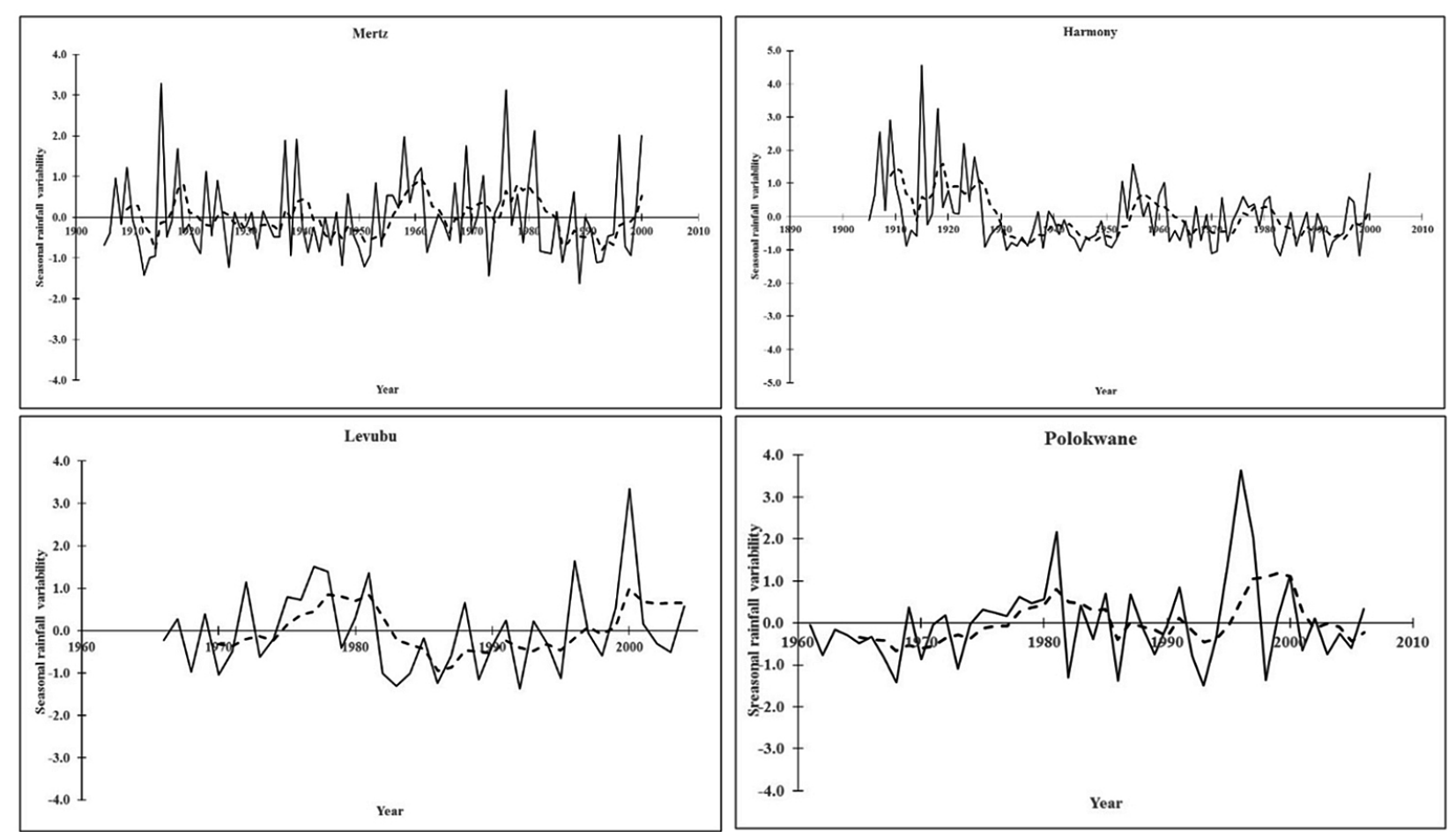

Figure 5. Seasonal rainfall variation at different locations in South Africa. Dotted line represents 5-year moving average.

\section{DISCUSSION}

\section{Frequency and trends of different daily rainfall amounts}

Daily rainfall was dominated by $<5 \mathrm{~mm}$ events under semiarid and sub-humid conditions of the selected locations of southern Africa. These rainfall quantities have a negligible effect on recharging soil moisture in soil types of southern Africa. Previous studies have shown that $20-25$ and $30-50 \mathrm{~mm}$ of rainfall are required to fully charge the top $30 \mathrm{~cm}$ of sandy and clay soils, respectively (Twomlow 1994; Twomlow and Bruneau, 2000). Most of the rainwater from such light showers can be lost through evaporation because of the high atmospheric evaporative demand (Barron, 2004). Some semi-arid areas experience 5$8 \mathrm{~mm} \cdot$ day $^{-1}$ evaporative water losses (Woltering, 2005) and less than $5 \mathrm{~mm} \cdot$ day $^{-1}$ showers are therefore insignificant for crop production. Under sub-humid conditions of southern Africa, atmospheric evaporative demand can reach $5 \mathrm{~mm} \cdot$ day $^{-1}$ during summer months (Trambauer et al. 2014), thereby making the light showers insignificant for cropping under such conditions.

Decreasing trajectories of 5-10 and, 11-20 mm.day ${ }^{-1}$ were detected at some locations in southern Africa. Rainfall amounts of 5-10 and, 11-20 mm.day ${ }^{-1}$ can have significant influence on crop growth, depending on soil and crop type, atmospheric evaporative demand and water management practices implemented in the cropping systems. When these rainfall amounts are received for 2 or 3 days, the soil profiles can be recharged with moisture, thereby facilitating crop growth. With the current trend of poor seasonal rainfall distribution and frequent in-crop dry spells (Ngetich et al., 2014), it is critical to capture this rainwater in order to prolong soil moisture availability in cropping systems. There were no linear trends in $>40 \mathrm{~mm} \cdot \mathrm{day}^{-1}$ at most of the selected locations. It is critical that rainwater from the few $40 \mathrm{~mm} \cdot \mathrm{day}^{-1}$ events be conserved through in-situ water capture practices (Mupangwa et al., 2007) or ex-situ storage for later use as supplementary irrigation (Rockström et al., 2003).

Despite the increased moisture stress associated with low rainfall of less than $5 \mathrm{~mm} \cdot \mathrm{day}^{-1}$, such amounts have sustained crop production across the region, though the yield gap is high.
Farmers have adapted to this through use of alternate cropping systems such as intercrops as well as shifting planting time. There is, however, still greater value from low rainfall events compared to the high rainfall events. Most farmers do not use in-situ moisture conservation; hence such amounts would not be very useful to the farmer given the fact that they occur at low frequencies. In addition, heavy rains are associated with challenges such as nutrient leaching, which increases fertilizer costs and ultimately reduces productivity (Geneti et al., 2019).

\section{Trends of monthly and seasonal rainfall}

Linear trends in monthly and seasonal rainfall varied with location, as some increasing and decreasing rainfall trajectories were indicated in the analyses conducted. This result is consistent with previous findings from studies conducted in southern Africa (Bellpart et al., 2015; Muthoni et al., 2019). Mitigation and adaptive measures to climate variability need to be informed by these local trends as blanket recommendations will not be effective. Significant seasonal rainfall increase occurred at semiarid Matopos station, and this is consistent with results from Muthoni et al. (2019), which revealed a 3-15 mm.year-1 increase in rainfall at some locations in south-western Zambia. Future rainfall projections have also indicated increases in rainfall in some parts of SSA (Shongwe et al., 2011).

Decreasing rainfall trends in some parts of southern Africa have been reported previously (Mason, 2001; Shi et al., 2007; Bellpart et al., 2015). These decreases in rainfall have been attributed to the influence of El Niño and shifts in atmospheric circulation processes (Nicholson et al., 2014; Gaughan et al., 2016). Smallholder farmers in parts of the region have generally observed declining rainfall over the years and acknowledge the importance of increasing adaptive measures in their farming systems (ZumaNetshiukwi et al., 2013; Mkuhlani et al., 2019). Significant rainfall decrease (up to $3.3 \mathrm{~mm} \cdot$ year $^{-1}$ ) in February rainfall occurred at a few of the locations. Differences in atmospheric drivers of rainfall patterns exist over short distances in southern Africa (Hachigonta and Reason, 2006; Manatsa and Matarira, 2009), and this could explain the variability between locations within the same agro- 
ecology. Additionally, Nicholson et al. (2014) reported that most of the inter-annual rainfall variability is generated during the March-April period of the growing season. The second half of the peak rainfall period is therefore at risk in southern Africa and cropping systems will continue experiencing soil moisture deficits at critical crop growth stages. The major food security cereals in southern Africa are sensitive to soil moisture deficits at reproductive growth stage which often occurs around December to February (Zaman-Allah, 2016), and this leads to significant yield reduction. Smallholder farming families would therefore be exposed to food deficits which are already prevalent in some parts of the region (FAO and ECA, 2018). Another related study, analysing optimum planting dates at Chitala and Chitedze in Malawi, confirmed a significant delay $(P<0.05)$ of 0.28 and 0.39 days.yr ${ }^{-1}$ in optimum planting dates at Chitala and Chitedze in Malawi, respectively, within the last 30 years, thereby making the length of the growing season increasingly shorter at these locations (Nyagumbo et al., 2017). Such changes in rainfall patterns over time corroborate findings from this study that the southern Africa region increasingly faces more difficult weather patterns for rainfed crop production.

Linear trends in monthly rainfall were location specific, a result that has been reported elsewhere in the region and for other SSA countries (Gummadi et al., 2017; Muthoni et al., 2019). Local factors such as topography or the presence of an inland water body can have a significant influence on spatial and temporal rainfall patterns (Goenster et al., 2015; Muthoni et al., 2019). The proximity of Chitala to Lake Malawi influenced the rainfall pattern and the location had greater chances of getting more rainfall than Chitedze and Dedza, which are located further inland in a relatively wetter agro-ecology. Muthoni et al. (2019) also reported the effects of local physical features such as mountains on spatiotemporal rainfall patterns in Tanzania. Adaptation strategies on smallholder farms in such areas with natural drivers of local rainfall patterns need to be tailor-made accordingly and cannot be generalized for the region.

\section{Seasonal rainfall variability}

The rainfall variability index (WMO, 1975) indicated the occurrence of drought conditions at the selected locations under different agro-ecological conditions. Droughts of varying degrees of severity occurred in $50 \%$ or more of the time periods considered in this study. Such drought frequency has been reported and is now a common phenomenon in southern Africa (Cooper et al., 2008; Nicholson et al., 2014; Bellprat et al., 2015). A drought frequency of every 3 to 4 years has been reported in some parts of southern Africa (World Bank, 2017). With such high frequencies, drought mitigation measures adapted to different biophysical and socio-economic smallholder farmer circumstances ought to be implemented to buffer cropping systems. Various adaptation and mitigation options have been developed and tested for smallholder conditions, and these include crop diversification (Twomlow et al., 2006), adapted crop types and varieties (Setimela et al., 2018), improving soil fertility (Zougmore et al., 2014), conservation agriculture-based practices (Thierfelder et al., 2017; Steward et al., 2018), and in-situ or ex-situ rainwater harvesting (Motsi et al., 2004; Mupangwa et al., 2007). Traditionally, droughts have been more severe in semi-arid areas (Graef and Haigis, 2001) and this is consistent with results from the low rainfall agro-ecologies of the current study, particularly Beitbridge in southern Zimbabwe. The importance of designing and implementing drought mitigation strategies cannot be over-emphasized in order to buffer rainfed farming systems. Climate-smart crop and livestock production practices are core for semi-arid areas and some adapted options are available for southern Africa (Chakoma et al., 2016; Thierfelder et al., 2017; Setimela et al., 2018).
Chitala location illustrated the influence of existing water bodies on local rainfall patterns in some parts of southern Africa. Despite this localized influence on rainfall, the threat of severe droughts was evident at Chitala and this highlights the importance of developing adaptation and mitigation interventions suited to local climatic conditions. All locations experienced incidences of either wet seasons followed immediately by mild to strong drought or the reverse trend. This has been occurring in southern Africa and is one of the major causes of chronic food shortages (Bell et al., 2003). Generally, the frequency of dry years increased between 1980 and 2007 compared to past years of 1950-1975 (Gaughan et al., 2016). This shift in southern Africa rainfall patterns and other climatic forcings has been reported previously (Manatsa and Behera, 2013; Nicholson et al., 2014; Bellprat et al., 2015), and emphasizes the need for climate-smart agricultural practices to buffer smallholder farming systems. The inter-seasonal rainfall variability has made planning and decision making on selection of crop species and cropping systems, and investments in agricultural inputs, difficult on smallholder farms.

\section{CONCLUSION}

Rainfall in southern Africa was dominated by $<5 \mathrm{~mm} \cdot$ day $^{-1}$ events in both semi-arid and sub-humid agro-ecological conditions. The frequency of 5-10 and 11-20 mm.day ${ }^{-1}$ varied with location, even where a large water body influenced the rainfall pattern. There were no apparent linear trends in monthly and seasonal rainfall at 15 of the 18 selected locations from southern Africa. Where trends were significant, a decreasing trajectory in February rainfall was detected at two locations. Increasing March and seasonal rainfall trajectories were apparent at a semi-arid location in southwestern Zimbabwe. Moderate and strong drought conditions were detected, and these also varied with location. Drought frequency was higher than $50 \%$, and a location close to a large water body also experienced strong drought conditions during the November-March growing season. All locations experienced incidences of a wet season followed immediately by very dry conditions, or vice versa, regardless of agro-ecological conditions.

Results of this study emphasize the need for policy to take due consideration of the prevailing climatic patterns in programming appropriate climate-smart adaptation measures that can help farmers to cope with the increasing frequency of droughts and ineffective rainfall events that make rainfed cropping riskier to farmers than it has been in the past 3 to 4 decades. It is also clear that policy makers need to invest more in reliable weather monitoring instruments so as to provide a higher density of measured weather patterns that help in informing the need for appropriate technological investments to cope with emerging weather patterns.

\section{ACKNOWLEDGEMENTS}

This study has been embedded into the CGIAR Research Programme MAIZE, Flagship Sustainable intensification of smallholder farming systems. We acknowledge the CGIAR Fund Council and other donors for funding to the CGIAR Research Programme MAIZE. We thank the meteorological stations from the four countries for their contribution in generating rainfall data used in the study.

\section{REFERENCES}

BARRON J (2004) Dry spell mitigation to upgrade semi-arid rainfed agriculture: Water harvesting and soil nutrient management for smallholder maize cultivation in Machakos, Kenya. PhD thesis, Department of Systems Ecology, Stockholm University, Sweden.

BELL M, GROVER M, HOOP M, KESTIN T, LYON B and SETH A (2003) IRI Climate Digest February 2002: Climate Impacts - January. URL: http://iri.columbia.edu/climate/cid/Feb2003/impacts/html 
BELLPRAT O, LOTT FC, GULIZIA C, PARKER HR, PUMPUCH LA, PINTO I, CIAVARELLA A and STOTT PA (2015) Unusual past dry and wet rainy seasons over Southern Africa and South America from a climate perspective. Weather Clim. Extremes. 9 36-46. https://doi. $\operatorname{org} / 10.1016 /$ j.wace.2015.07.001

BEST DJ and GIPPS PG (1974) Algorithm AS 71: The upper tail probabilities of Kendall's tau. R. Stat. Soc. 23 (1) 98-100. https://doi. org/10.2307/2347062

BURN DH and ELNUR MAH (2002) Detection of hydrologic trends and variability. J. Hydrol. 255 (1-4) 107-122. https://doi.org/10.1016/ S0022-1694(01)00514-5

CHAKOMA I, MANYAWU G, GWIRIRI LC, MOYO S, DUBE S, IMBAYARWO-CHIKOSI VE, HALIMANI T, CHAKOMA C, MAASDORP BV and BUWU V (2016) Promoting the use of homemixed supplements as alternatives to commercial supplements in smallholder beef production systems in the sub-humid region of Zimbabwe. Afr. J. Range Forage Sci. 33 (3) 165-171. https://doi.org/1 $0.2989 / 10220119.2016 .1207706$

CONWAY D, VAN GARDEN EA, DERYNG D, DORLING S, KRUEGER T, LANDMAN W, LANKFORD B, LEBEK K, ASBORN T, RINGLER C, THURLOW J, ZGU T and DALIN C (2015) Climate and southern Africa's water-energy-food nexus. Nat. Clim. Change. 5 837-846. http://dx.doi.org/10.1038/NCLIMATE2735

COOPER PJM, DIMES J, RAO KPC, SHAPIRO B, SHEIFERAW B and TWOMLOW S (2008) Coping better with current climatic variability in the rain-fed farming systems of sub-Saharan Africa: an essential first step in adapting to future climate change? Agric. Ecosyst. Environ. 126 24-35. https://doi.org/10.1016/j.agee.2008.01.007

DARRON JD (2014) Regional Climate messages: Southern Africa Scientific report from the CARIAA Adaptation at Scale in SemiArid Regions (ASSAR) Project, December 2014.

DA SILVA RM, SANTOS CA, MOREIRA M, CORTE-REAL J, SILVA VC and MEDEIROS IC (2015) Rainfall and river flow trends using Mann-Kendall and Sen's slope estimator statistical tests in the Cobres River basin. Nat. Hazards. 77 (2) 1205-1221. https://doi. org/10.1007/s11069-015-1644-7

DIXIT PN, COOPER PJM, DIMES J and RAO KPC (2011) Adding value to field based agronomic research through climate risk assessment A case study of maize production in Kitale, Kenya. Exp Agric. 47 317-338. https://doi.org/10.1017/S0014479710000773

FAO and ECA (2018) Regional overview of food security and nutrition. Addressing the threat from climate variability and extremes for food security and nutrition. Accra. $116 \mathrm{pp}$.

FANADZO M and NCUBE B (2017) Challenges and opportunities for revitalising smallholder irrigation schemes in South Africa. Water SA. 44 (3) 436-447. https://doi.org/10.4314/wsa.v44i3.11

GAUGHAN AE, STAUB CG, HOELL A, WEAVER A and WAYLEN PR (2016) Inter and intra-annual precipitation variability and associated relationships to ENSO and the IOD in southern Africa. Int. J. Clim. 36 1643-1656. https://doi.org/10.1002/joc.4448

GENETI TZ (2019) Review on the effect of moisture or rain fall on crop production. Civ. Environ. Res. 11 (2). https://doi.org/10.7176/ CER/11-2-01

GOENSTER S, WIEHLE M, GEBAUER J, ALI AM and STERN RD (2015) Daily rainfall data to identify trends in rainfall amount and rainfall-induced agricultural events in the Nuba Mountains of Sudan. J Arid Environ. 122 16-26. https://doi.org/10.1016/j. jaridenv.2015.06.003

GRAEF F and HAIGIS J (2001) Spatial and temporal rainfall variability in the Sahel and its effects on farmers' management strategies. J Arid Environ. 48 221-231. https://doi.org/10.1006/jare.2000.0747

GUMMADI S, RAO KPC, SEID J, LEGESSE G, KADIYALA MDM, TAKELE R, AMEDE T and WHITBREAD A (2017) Spatio-temporal variability and trends of precipitation and extreme rainfall events in Ethiopia in 1980-2010. Theor. Appl. Climatol. 134 1315-1328. https://doi.org/10.1007/s00704-017-2340-1

HACHIGONTA S and REASON CJC (2006) Interannual variability in dry and wet spell characteristics over Zambia. Clim. Res. 32 49-62. https://doi.org/10.3354/cr032049

KARMESHU N (2012) Trend detection in annual temperature and precipitation using the Mann Kendall test - a case study to assess climate change on selected states in the Northeastern United States. University of Pennsylvania, Pennsylvania.
KENDALL MG (1975) Rank Correlation Methods (2 $2^{\text {nd }}$ edn). Hafner, New York.

LOBELL DB, BURKE MB, TEBALDI C, MASTRANDREA MD, FALCON WP and NAYLOR RL (2008) Prioritizing climate change adaptation needs for food security in 2030. Science. 319 607-610. https://doi.org/10.1126/science.1152339

LOVE D, UHLENBROOK S, TWOMLOW S and VAN DER ZAAG P (2010) Changing rainfall and discharge patterns in the northern Limpopo Basin, Zimbabwe. Water SA. 36 (3) 335-350.

MAMOMBE V, KIM W and CHOI Y (2017) Rainfall variability over Zimbabwe and its relation to large-scale atmosphere-ocean processes. Int. J. Climatol. 37 963-971. https://doi.org/10.1002/joc. 4752

MANATSA D and MATARIRA CH (2009) Changing dependence of rainfall variability on ENSO and the Indian dipole/zonal mode. Theor. Appl. Climatol. 98 375-396. https://doi.org/10.1007/s00704009-0114-0

MANATSA D and BEHERA SK (2013) On the major shifts in the IOD during the last century, the role of the Mascarene High displacements. Int. J. Climatol. 34 (6) 2033-2046. https://doi.org/ 10.1002/joc. 3820

MANYAWU G, GWIRIRI LC, CHAKOMA I, MOYO S and DUBE S (2016) The effect of conditioning and natural aeration methods on rate of moisture loss and crude protein content of Lablab purpureus herbage during hay-making. Afr. J. Range Forage Sci. 33 (4) 247-251. https://doi.org/10.2989/10220119.2016.1256349

MASIAH I, MASKEY S, MUSSA FEF and TRAMBAUER P (2014) A review of droughts on the African continent: a geospatial and longterm perspective. Hydrol. Earth Syst. Sci. 18 3635-3649. https://doi. org/10.5194/hess-18-3635-2014

MASON SJ (2001) El Nino, climate change and southern African climate. Environ. 12 619-638. https://doi.org/10.1002/env.476

MAURE G, PINTO I, NDEBELE-MURISA M, MUTHIGE M, LENNARD C, NIKULIN G and DOSIO A (2018) The southern African climate under $1.5^{\circ} \mathrm{C}$ and $2{ }^{\circ} \mathrm{C}$ of global warming as simulated by CORDEX regional climate models. Environ. Res. Lett. 13065002. https/doi.org/10.1088/1748-9326/aab190

MKUHLANI S, CRESPO O, RUSERE F and ZHOU L (2019) Classification of small-scale farmers for improved rainfall variability management in South Africa. Agric. Sust.Food Syst. 44 (1) 7-29. https://doi.org/10.1080/21683565.2018.1537325

MOTSI KE, CHUMA E and MUKAMURI BB (2004) Rainwater harvesting for sustainable agriculture in communal lands of Zimbabwe. Phys. Chem. Earth. 29 1069-1073. https://doi.org/10. 1016/j.pce.2004.08.008

MUPANGWA W, TWOMLOW S, WALKER S and HOVE L (2007) Effect of minimum tillage and mulching on maize (Zea mays L.) yield and water content of clayey and sandy soils. Phys. Chem. Earth. 32 1127-1134. ttps://doi.org/10.1016/j.pce.2007.07.030

MUPANGWA W, WALKER S and TWOMLOW S (2011) Start, end and dry spells of the growing season in semi-arid southern Zimbabwe. J Arid Environ. 75 1097-1104. https://doi.org/10.1016/j. jaridenv.2011.05.011

MUTHONI FK, ODONGO VO, OCHIENG J, MUGALAVAI EM, MOURICE SK, HOESCHE-ZELEDON I, MWILA $M$ and BEKUNDA M (2019) Long term spatial-temporal trends and variability of rainfall over Eastern and Southern Africa. Theor. Appl. Climatol. 139 1869-1882. https://doi.org/10.1007/s00704-018-2712-1

NGETICH KE, MUCHERU-MUNA M, MUGWE JN, SHISANYA CA, DIELS J and MUGENDI DN (2014) Length of growing season, rainfall temporal distribution, onset and cessation dates in the Kenyan highlands. Agric. Forest Meteorol. 188 24-32. https://doi. org/10.1016/j.agrformet.2013.12.011

NICHOLSON SE, KLOTTER D and CHAVULA G (2014) A detailed rainfall climatology for Malawi, Southern Africa. Int. J. Climatol. 34 315-325. https://doi.org/10.1002/joc.3687

NYAGUMBO I, MKUHLANI S, MUPANGWA W and RODRIGUEZ D (2017) Planting date and yield benefit from conservation agriculture across southern Africa. Agric Syst. 150 21-33. https:// doi.org/10.1016/j.agsy.2016.09.016

OGENGA JO, MUGALAVAI EM, NICODEMUS O and NYANDIKO NO (2018) Impact of rainfall variability on food production under rainfed agriculture in Homa Bay County, Kenya. Int. J. Sci. Res. Publ. 8 (8) 857-870. https://doi.org/10.29322/IJSRP.8.8.2018.p80110 
POHLERT T (2018) Non-parametric trend tests and change-point detection. R Package Version 1.1.1. URL: https://cran.r-project.org/ web/packages/trend/vignettes/trend.pdf.

PRASANNA BM, HUESING JE, EDDY R and PESCHKE VM (eds) (2018) Fall Armyworm in Africa: A Guide for Integrated Pest Management ( $1^{\text {st }}$ edn). CDMX: CIMMYT, Mexico.

R CORE TEAM (2018) R: A language and environment for statistical computing. R Foundation for Statistical Computing, Vienna. URL: https://www.R-project.org

ROCKSTROM J, BARRON J and FOX P (2003) Water productivity in rain-fed agriculture: challenges and opportunities for smallholder farmers in drought-prone tropical agroecosystems. In: Kijne JW, Barker R and Molden D (eds) Water Productivity in Agriculture: Limits and Opportunities for Improvement. CAB International Delft. 145-162. https://doi.org/10.1079/9780851996691.0145

SADC (Southern African Development Community) (2016) SADC Regional Vulnerability Assessment and Analysis Synthesis Report 2016. August 2016, Pretoria, South Africa. URL: https://reliefweb. int > files > resources > SADC_RVAA-August-Final-Web (Accessed 24 September 2019).

SETIMELA P, GASURA E, THIERFELDER C, ZAMAN-ALLAH M, CAIRNS J and BODDUPALLI PM (2018) When the going gets tough: Performance of stress tolerant maize during the 2015/16 (El Niño) and 2016/17 (La Niña) season in southern Africa. Agric. Ecosyst. Environ. 268 79-89. https://doi.org/10.1016/j.agee.2018.09.006

SHI G, RIBBIE J, CAI W and COWAN T (2007) Multidecadal variability in the transmission of ENSO signals to the Indian Ocean. Geophys. Res. Lett. 34 L09706. https://doi.org/10.1029/2007GL029528

SHONGWE ME, VAN OLDENBORGH GJ and VAN DEN HURK BJJM (2009) Projected changes in mean and extreme precipitation in africa under global warming. Part I: Southern Africa. J. Clim. 22 (13) 3819-3837. https://doi.org/10.1175/2009JCLI2317.1

SHONGWE ME, VAN OLDENBORGH GJ and VAN DEN HURK BJJM (2011) Projected changes in mean and extreme precipitation in Africa under global warming. Part II: East Africa. J Clim. 24 37183733. https://doi.org/10.1175/2010JCLI2883.1

SILESHI G, AKNIFESI FK, DEBUSHO LK, BEEDY T, AJAYI OC and MONGOMBA S (2009) Variation in maize yield gaps with plant nutrient inputs, soil type and climate across sub-Saharan Africa: A Review. Field Crops Res. https://doi.org/10.1016/j.fcr.2009.11.014

SMALE M, BYERLEE D and JAYNE T (2011) Maize revolutions in subSaharan Africa. In an African green revolution. Springer, Dordrecht. 165-195. https://doi.org/10.1596/1813-9450-5659

SONWA DJ, DIEYE A, EL MZOURI EH, MAJULE A, MUGABE FT, OMOLO N, WOUAPI H, OBANDO J and BROOKS N (2017) Drivers of climate risk in African agriculture. Clim. Dev. 9 (5) 383 398. https://doi.org/10.1080/17565529.2016.1167659

STEWARD PR, THIERFELDER C, DOUGILL AJ and LIGOWE I (2018) Conservation agriculture enhances resistance of maize to climate stress in a Malawian medium-term trial. Agric. Ecosyst. Environ. 277 95-104. https://doi.org/10.1016/j.agee.2018.07.009

TADROSS M, HEWTSON BC and USMAN MT (2005) The interannual variability of onset of the maize growing season over South Africa and Zimbabwe. J Clim. 18 3356-3372. https://doi.org/10.1175/JC LI3423.1

THIERFELDER C, CHIVENGE P, MUPANGWA W, ROSENSTOCK TS, LAMANNA C and EYRE JX (2017) How climate-smart is conservation agriculture (CA)? - its potential to deliver on adaptation, mitigation and productivity on smallholder farms in southern Africa. Food Security. 9 537-560. https://doi.org/10.1007/ s12571-017-0665-3

THIERFELDER C, BAUDRON F, SETIMELA P, NYAGUMBO I, MUPANGWA W, MHLANGA B, LEE N and GERARD B (2018) Complementary practices supporting conservation agriculture in southern Africa. A review. Agron. Sust. Dev. 38 Article 16. https:// doi.org/10.1007/s13593-018-0492-8
TRAMBAUER P, DUTRA E, MASKEY S, WERNER M, PAPPENBERGER F, VAN BEEK LPH and UHLENBROOK $S$ (2014) Comparison of different evaporation estimates over the African continent. Hydrol. Earth Syst. Sci. 18 193-212. https://doi. org/10.5194/hess-18-193-2014

TWOMLOW SJ (1994) Field moisture characteristics of two fersiallitic soils in Zimbabwe. Soil Use Manage. 10 168-173. https://doi. org/10.1111/j.1475-2743.1994.tb00481.x

TWOMLOW SJ and BRUNEAU PMC (2000) The influence of tillage on semiarid soil-water regimes in Zimbabwe. Geoderma. 95 33-51. ttps://doi.org/10.1016/S0016-7061(99)00071-3

TWOMLOW SJ, STEYN DU PREEZ JT and DU PREEZ CC (2006) Dryland farming in southern Africa. In: Petersen GA, Unger WP, Payne WA (eds) Dryland Agriculture ( $\left.2^{\text {nd }} \mathrm{edn}\right)$. Agronomy Monograph No. 23 American Society of Agronomy, Madison, Wisconsin. 769836. https://doi.org/10.2134/agronmonogr23.2ed.c19

USMAN MT and REASON CJC (2004) Dry spell frequencies and their variability over southern Africa. Clim. Res. 26 199-211. https://doi. org/10.3354/cr026199

VAN ITTERSUM MK, CASSMAN KG, GRASSINI P, WOLF J, TITONELL P and HOCHMAN Z (2013) Yield gap analysis with local and global relevance - A review. Field Crops Res. 143 4-17. https://doi.org/10.1016/j.fcr.2012.09.009

WANG XL and FENG Y (2013) RH tests V4 User Manual Climate Research Division. Atmospheric Science and Technology Directorate, Science and Technology Branch, Environment Canada. URL: http://etccdi.pacificclimate.org/software.shtml. 28 pp.

WOLTERING L (2005) Estimating the influence of on-farm conservation practices on the water balance: Case of the Mzinyathini catchment in Zimbabwe. MSc. thesis. Delft University of Technology, The Netherlands. 103 pp.

WORLD BANK (2017) Climate Change Knowledge Portal. World Bank. http://sdwebx.worldbank.org/climateportal/index.cfm?page= country_vulnerabilities\&ThisRregion+Africa\&ThisCcode $=\mathrm{MOZ}$

WMO (World Meteorological Organization) (1975) Drought and Agriculture. WMO/TN 138. WMO, Geneva.

ZAIDI PH, ZAMAN-ALLAH M, TRASCHEL S, SEETHARAM K, CAIRNS JE and VINAYAM MT (2016) Phenotyping for abiotic stress tolerance in maize - heat stress. A Field Manual. CIMMYT: Hyderabad, India. 32 pp.

ZAMAN-ALLAH M, ZAIDI PH, TRASCHEL S, CAIRNS JE, VINAYAN MT and SEETHARAM K (2016) Phenotyping for abiotic stress tolerance in maize - drought stress. A Field Manual. CIMMYT, Mexico. 32 pp.

ZINYENGERERE N, MHIZHA T, MASHONJOWA E, CHIPINDU B, GEERTS S and RAES D (2011) Using seasonal climate forecasts to improve maize production decision support in Zimbabwe. Agric. For. Meteorol. 151 (12) 1792-1799. https://doi.org/10.1016/j. agrformet.2011.07.015

ZOUGMORE R, JALLOH A and TIORO A (2014) Climate-smart soil water and nutrient management options in semi-arid West Africa: a review of evidence and analysis of stone bunds and zaï techniques. Agric. Food Security. 3 16. https://doi.org/10.1186/2048-7010-3-16

ZUMA-NETSHIUKHWI G, STIGTER K and WALKER S (2013) Use of traditional weather/climate knowledge by farmers in the southwestern Free State of South Africa: agrometeorological learning by scientists. Atmosphere. 4 383-410. https://doi.org/10.3390/ atmos 4040383 
APPENDIX

Table A1. Locations and years considered for different meteorological stations used in the rainfall analyses

\begin{tabular}{|c|c|c|c|c|c|}
\hline Country & Station & Years & Latitude & Longitude & Altitude (m) \\
\hline \multirow[t]{6}{*}{ Zimbabwe } & Harare & 1963-2001 & -17.72 & 31.02 & 1475 \\
\hline & Marondera & $1952-2000$ & -18.93 & 31.54 & 1658 \\
\hline & Bulawayo & 1931-2001 & -20.16 & 28.61 & 1356 \\
\hline & Matopos & 1940-2015 & -20.51 & 28.44 & 1347 \\
\hline & West Nich. & $1963-2001$ & -21.06 & 29.36 & 864 \\
\hline & Beitbridge & 1952-2001 & -22.21 & 29.99 & 462 \\
\hline \multirow[t]{3}{*}{ Malawi } & Chitala & 1948-1999 & -13.68 & 34.25 & 606 \\
\hline & Chitedze & 1981-2013 & -13.98 & 33.64 & 1100 \\
\hline & Dedza & 1959-1999 & -14.32 & 34.25 & 1632 \\
\hline \multirow[t]{5}{*}{ Mozambique } & Chimoio & 1952-2012 & -19.25 & 33.43 & 693 \\
\hline & Chokwe & 1962-1999 & -24.53 & 32.98 & 33 \\
\hline & Pemba & 1952-2005 & -12.59 & 40.52 & 70 \\
\hline & Quelimane & 1961-2008 & -17.86 & 36.87 & 5 \\
\hline & Xai Xai & 1952-1989 & -25.09 & 33.53 & 2 \\
\hline \multirow[t]{4}{*}{ South Africa } & Harmony & 1905-2000 & -23.08 & 29.85 & 517 \\
\hline & Levubu & 1966-2004 & -23.08 & 30.28 & 706 \\
\hline & Mertz & 1905-2000 & -26.50 & 28.36 & 1521 \\
\hline & Polokwane & 1961-2006 & -23.73 & 29.59 & 1194 \\
\hline
\end{tabular}

